\title{
Lymphatic impairment leads to pulmonary tertiary lymphoid organ formation and alveolar damage
}

\author{
Hasina Outtz Reed, ${ }^{1,2}$ Liqing Wang, ${ }^{3}$ Jarrod Sonett, ${ }^{4}$ Mei Chen, ${ }^{2}$ Jisheng Yang, ${ }^{2}$ Larry Li, ${ }^{2}$ Petra Aradi, $, 5,6$ Zoltan Jakus, \\ Jeanine D'Armiento, ${ }^{4}$ Wayne W. Hancock, ${ }^{3}$ and Mark L. Kahn ${ }^{2}$ \\ 'Department of Medicine and Division of Pulmonary and Critical Care, ${ }^{2}$ Department of Medicine and Cardiovascular Institute, University of Pennsylvania, Philadelphia, Pennsylvania, USA. ${ }^{3}$ Department of \\ Pathology and Laboratory Medicine, Division of Transplant Immunology, Children's Hospital of Philadelphia and University of Pennsylvania, Philadelphia, Pennsylvania, USA. ${ }^{4}$ Department of Anesthesiology, \\ Center for Molecular Pulmonary Disease, College of Physicians and Surgeons, Columbia University, New York, New York, USA. ${ }^{5}$ Department of Physiology, Semmelweis University School of Medicine, \\ Budapest, Hungary. ${ }^{6}$ MTA-SE "Lendület" Lymphatic Physiology Research Group of the Hungarian Academy of Sciences and the Semmelweis University, Budapest, Hungary.
}

\begin{abstract}
The lung is a specialized barrier organ that must tightly regulate interstitial fluid clearance and prevent infection in order to maintain effective gas exchange. Lymphatic vessels are important for these functions in other organs, but their roles in the lung have not been fully defined. In the present study, we evaluated how the lymphatic vasculature participates in lung homeostasis. Studies using mice carrying a lymphatic reporter allele revealed that, in contrast to other organs, lung lymphatic collecting vessels lack smooth muscle cells entirely, suggesting that forward lymph flow is highly dependent on movement and changes in pressure associated with respiration. Functional studies using C-type lectin domain family 2-deficient (CLEC2deficient) mice in which lymph flow is impaired because of loss of lympho-venous hemostasis, or using inducible lungspecific ablation of lymphatic endothelial cells in a lung transplant model revealed that loss of lymphatic function leads to an inflammatory state characterized by the formation of tertiary lymphoid organs (TLOs). In addition, impaired lymphatic flow in mice resulted in hypoxia and features of lung injury that resembled emphysema. These findings reveal both a lung-specific mechanism of lymphatic physiology and a lung-specific consequence of lymphatic dysfunction that may contribute to chronic lung diseases that arise in association with TLO formation.
\end{abstract}

\section{Introduction}

The lymphatic vascular system transports fluid, immune cells, and lipids throughout the body to prevent tissue edema, facilitate adaptive immune responses, and enable efficient fat handling. The lymphatic network is typically depicted as a network of smaller lymphatic capillaries that are specialized to take up cells, protein, and fluid, as well as larger collecting lymphatics that are designed to return lymph to the venous system and facilitate immune surveillance in secondary lymphoid organs such as lymph nodes (LNs). Recent studies of the blood vascular system have highlighted molecular and structural features that contribute to organ-specific roles of these vessels in the blood-brain barrier, liver sinusoids, and the bone marrow hematopoietic niche (1). With the exception of villus lacteals, which are specialized for the uptake and transport of dietary fats, the extent to which the lymphatic vascular system exhibits a similar organ-specific structure and function at other sites in the body remains unclear.

Lymphatic vessels are relatively abundant in the lung, an organ that is uniquely sensitive to edema and inflammation, which may impair gas exchange. In the developing lung, lymphatic function is required to drain fluid and achieve the compliance that is nec-

Conflict of interest: The authors have declared that no conflict of interest exists. Copyright: $\odot$ 2019, American Society for Clinical Investigation.

Submitted: September 20, 2018; Accepted: April 2, 2019; Published: May 20, 2019

Reference information: J Clin Invest. 2019;129(6):2514-2526.

https://doi.org/10.1172/JCl125044. essary for neonatal lung inflation $(2,3)$, but the role of lymphatics in the mature lung is undefined. One proposed function of pulmonary lymphatics is to drain fluid from the lung in order to prevent pulmonary edema that would reduce gas exchange. However, in the canonical physiologic model proposed by Ernest Starling, fluid balance in the lung is maintained by a balance of hydrostatic forces that move fluid from the blood and into the interstitium with oncotic forces that move fluid from the interstitium into the blood. In this model, lymphatics are not required to maintain fluid balance, but the role of pulmonary lymphatics in lung fluid homeostasis has yet to be fully tested. Short-term studies using catheter-based approaches in large animals have attempted to investigate the function of lymphatics in the lung (4), but lymph flow is exceedingly slow compared with flow in the blood circulation $(2 \mathrm{ml} / \mathrm{min}$ versus $5000 \mathrm{ml} / \mathrm{min}$ ) (5). Thus, the consequences of changes in lymphatic function may require days to weeks to manifest.

The lung is constantly exposed to the outside environment and must maintain both a quiescent immune state while having the ability to generate a robust immune response to pathogens in order to prevent infection. Immune cell trafficking to draining LNs via the pulmonary lymphatics plays a central role in coordinating the adaptive immune response to infection and other pathogens (6, 7). Chronic inflammation is often associated with the development of tertiary lymphoid organs (TLOs), also known as inducible bronchus-associated lymphoid tissue (iBALT), which are accumulations of lymphoid cells that resemble LNs in cellular content, organization, and the presence of lymphatic vessels $(8,9)$. Although TLOs 
Prox1-EGFP SMA
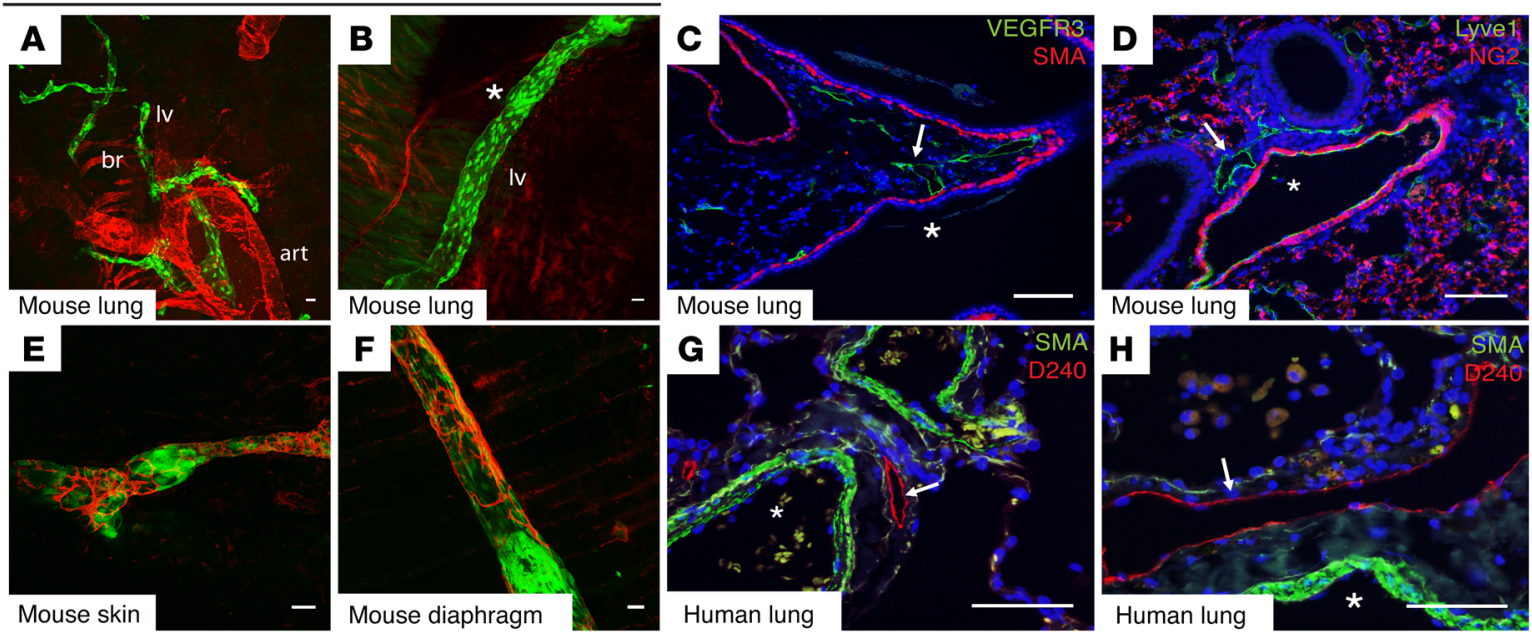

Figure 1. Pulmonary collecting lymphatics lack SMC or pericyte coverage. (A and B) Whole-mount images of lungs from adult Prox1-EGFP lymphatic reporter mice show pulmonary lymphatic vessels (Iv) in green, with the asterisk in $\mathbf{B}$ indicating an area of Prox ${ }^{\text {hi }}$ endothelial cells that marks lymphatic valves. Note the staining for SMA (red) present on both the bronchi (br) and arteries (art). (C and D) Immunohistochemical analysis of lung sections shows pulmonary lymphatics in green using staining for VEGFR3 or Lyve1 (arrows). Staining for SMA (C, red) or NG2 (D, red) marks airways and blood vessels, respectively. Asterisks indicate the large airway (C) and blood vessel (D) in proximity to lymphatic vessels. (E and $\mathbf{F}$ ) Whole-mount staining for SMA (red) on lymphatic vessels in skin (E) and diaphragm (F) from Prox1-EGFP mice. (G and $\mathbf{H})$ Human lung tissue sections were stained for the lymphatic molecular marker PDPN using the D240 antibody (red, arrows) and SMA (green), with arteries indicated by asterisks. Scale bars: $25 \mu \mathrm{m}$.

are a hallmark of chronic lung disease (10), it is unclear why they result from such widely differing insults such as chronic cigarette smoke exposure and infection. Although lymphatic vessels and lymphangiogenesis are key features of TLOs $(8,11)$, whether TLO formation is connected to lymphatic function is unknown. Finally, although abnormal lymphatics have been described in association with a variety of lung diseases (12), whether they are simply a consequence of the disease or whether lymphatic dysfunction actively contributes to lung injury is unknown.

To address the role of lymphatics in the adult lung, we have characterized pulmonary lymphatic anatomy and used 2 distinct models of impaired pulmonary lymphatic flow in the mouse: C-type lectin domain family 2-deficient (CLEC2-deficient) mice in which blood obstructs the forward flow of lymph in the lung (13), and mice in which the pulmonary lymphatics have been genetically engineered to enable specific deletion using diphtheria toxin following lung transplantation. We found that pulmonary collecting lymphatics have valves but lack smooth muscle cells (SMCs), a unique characteristic of these vessels that is consistent with propulsion of lymph through respiration-associated changes in thoracic pressure rather than contraction of the vessel. Functional studies demonstrate that mice with impaired pulmonary lymphatic flow are susceptible to increased pulmonary edema following lung injury and develop pronounced leukocyte accumulation and TLO formation in the lung parenchyma, even in the absence of injury. Unexpectedly, we found that mice with TLO formation associated with impaired lymphatic flow developed hypoxia and lung injury, with several features of human emphysema. These findings identify unique physiological and pathological aspects of lymphatic function in the lung and suggest that lymphatic dysfunction may play a role in the pathogenesis of chronic lung disease.

\section{Results}

Pulmonary collecting lymphatics lack SMC coverage. To address the specific roles of lymphatic vessels in pulmonary physiology and pathophysiology, we first carefully examined lung lymphatic vascular anatomy. The lymphatic system classically consists of both smaller primary lymphatic capillaries that take up fluid, proteins, and cells from the tissue as well as larger collecting vessels that transport lymph to LNs and, ultimately, the venous system. Whereas lymph flow within lymphatic capillaries is passive, the collecting lymphatics are typically characterized by the presence of functional units known as lymphangions that actively pump lymph (14). Lymphangions consist of SMC-lined segments of lymphatic vessel separated by valves (15). Physiologic studies of collecting lymphatics from the limb and mesentery have demonstrated that active SMC contraction and valve activity are coordinated to create waves of forward movement of lymph (16-19). In the lung, however, physiologic studies using catheter-based measurement of pulmonary lymph flow in anesthetized large animals have suggested that extrinsic forces such as changes in thoracic pressure associated with respiration may play a more central role (3, 4, 20-22).

To characterize the pulmonary lymphatic network and to compare it with better-characterized lymphatic beds such as those in the gut and mesentery, we performed whole-mount imaging of lungs from Prox1-EGFP mice in which lymphatic endothelial cells (LECs) are marked by GFP expression (23). Whole-mount immunostaining for smooth muscle actin (SMA) revealed complete coverage of arterial vessels and characteristic partial coverage of bronchi, but virtually no SMA staining was detected on pulmonary lymphatic vessels (Figure 1, A and B). Even the largest lymphatic collecting vessels in the lung, identified by their more proximal location adjacent to large airways and blood vessels (Figure 1A) as well as by the presence of Prox $1^{\text {hi }}$ endothelial cells that mark 


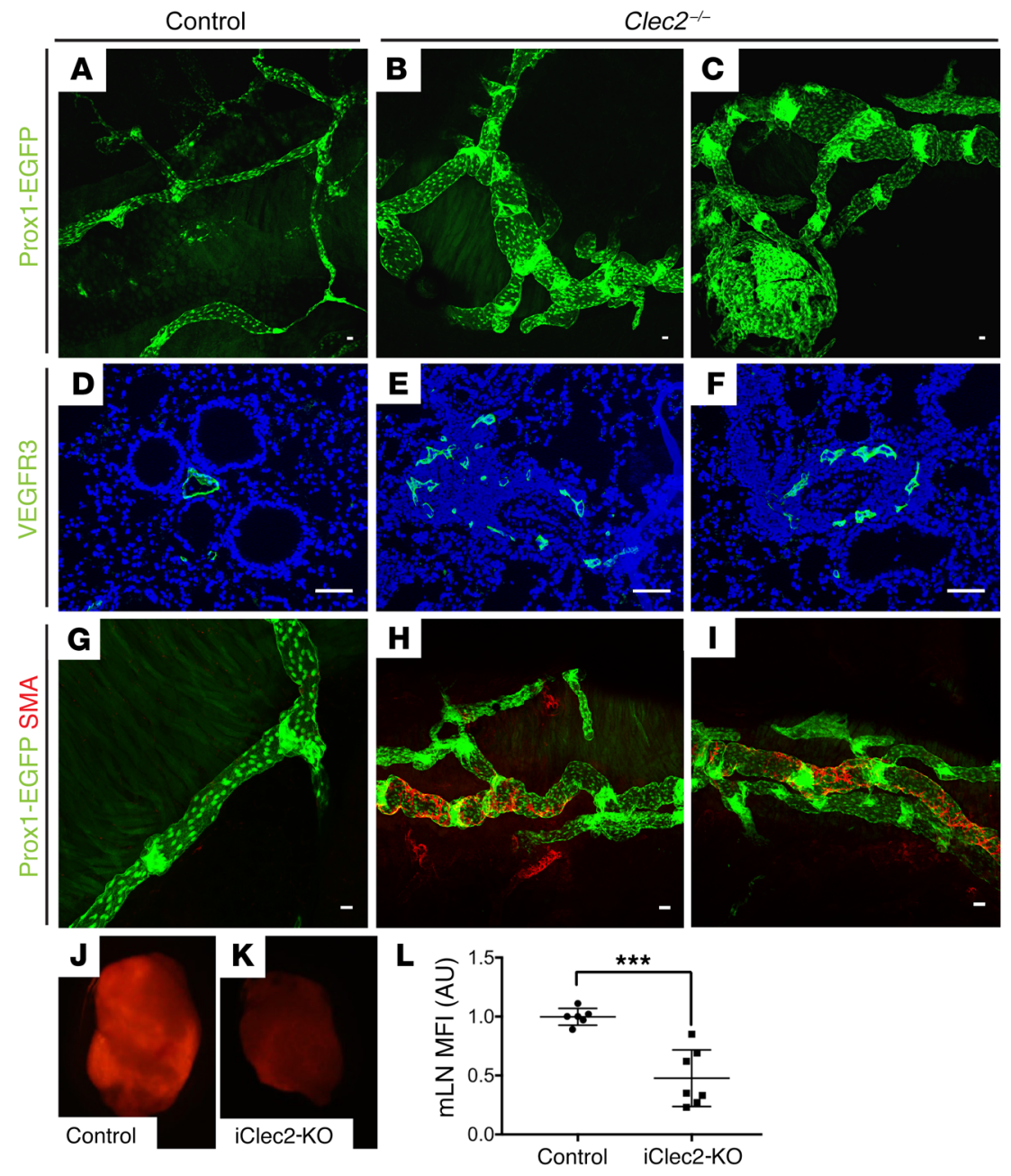

Figure 2. CLEC2-deficient mice exhibit impaired lymphatic flow. (A-C) Whole-mount images of pulmonary lymphatic vessels (green) using the lymphatic reporter Prox1-EGFP in 4- to 8-week-old $\mathrm{Clec}^{-/-}$and control mice. (D-F) Immunohistochemical analysis of lung sections stained for VEGFR3 (green) shows lymphatic vessels of 4- to 8-week-old $\mathrm{Clec}^{-/-}$and control mice. (G-I) Whole-mount SMA-stained (red) images of pulmonary lymphatic vessels (Prox1-EGFP, green) from 4- to 8-week-old Clec2 ${ }^{-/-}$and control mice. (J and $\mathbf{K}$ ) Fluorescence microscopic images of $\mathrm{mLNs}$ from iClec2-KO and control mice 50 minutes after intratracheal administration of dextran-568 (red). (L) Quantification of dextran-568 uptake into $\mathrm{mLNs}$ by MFI (AU). Scale bars: $25 \mu \mathrm{m}$. Data are representative of at least 5 mice in each group. All values represent the mean \pm SEM. ${ }^{* * *} P<0.001$, by Student's $t$ test.

lymphatic valves (Figure 1B), were devoid of all SMA staining. Using conventional immunohistochemical analyses of lung sections, SMA staining could be seen lining airways, but neither SMA nor the pericyte marker NG2 was detectable alongside lymphatic endothelium marked by VEGFR3 or Lyve1 expression (Figure $1, C$ and D). Prior studies have reported SMA staining alongside Lyve $1^{+}$endothelial cells (24), but careful analysis of Lyve1 staining revealed that, unlike VEGFR3 staining, Lyve1 was readily detected in large blood vessels and capillaries in the lung and therefore did not independently serve as a specific marker of lung lymphatic vessels (Figure 1, C and D and Supplemental Figure 1; supplemental material available online with this article; https://doi.org/10.1172/ JCI125044DS1). Analysis of 180 distinct lung lymphatic vessels identified using VEGFR3 staining from 6 different animals revealed no associated $\mathrm{SMA}^{+}$cells (Supplemental Table 1). In contrast, collecting lymphatics in other tissues such as the skin (Figure $1 \mathrm{E}$ ) and diaphragm (Figure $1 \mathrm{~F}$ ) showed robust SMC coverage in nonvalvular regions, consistent with classic lymphangion anatomy $(16,19)$. Importantly, immunostaining of collecting lymphatic vessels in normal human lung tissue, identified by expression of the lymphatic endothelium-specific marker podoplanin (PDPN) $(25,26)$, also showed a lack of lymphatic SMC coverage (Figure 1, $\mathrm{G}$ and $\mathrm{H}$ ). These results reveal that collecting lymphatic vessels in the lung have a unique anatomy in which the classic lymphangion is absent and suggest that lymph flow in the lung does not rely on intrinsic pumping of the collecting lymphatic vasculature.

Mice lacking CLEC2 have abnormal pulmonary lymphatic morphology and function. To functionally address the role of lymphatic vessels in the mature lung over time, we studied animals lacking the CLEC2 receptor (27). CLEC2 is expressed predominantly on platelets, and its activation by PDPN expressed on the surface of lymphatic but not blood endothelial cells is required to maintain separation of the venous and lymphatic vasculature through formation of a platelet plug at the lympho-venous junction $(13,27$, 28). In the absence of CLEC2, there is chronic retrograde flow of blood from the higher-pressure venous system into the thoracic duct that impairs forward lymph flow (29). Given the close physical proximity of the lympho-venous junction to the site at which the pulmonary lymphatic vasculature drains into the thoracic duct, we hypothesized that lymph flow in pulmonary lymphatic vessels in Clec2-mutant mice would be significantly compromised. As previously observed in mesenteric lymphatics (29), we observed that loss of CLEC2 resulted in dilated and tortuous pulmonary lymphatic vessels (visualized using the Prox1-EGFP transgene and immunostaining for VEGFR3) compared with that seen in controls by 4 weeks of age (Figure 2, A-F). We have previously found that impaired lymph flow due to loss of CLEC2 is associated with abnormal mesenteric collecting lymphatic vessel 

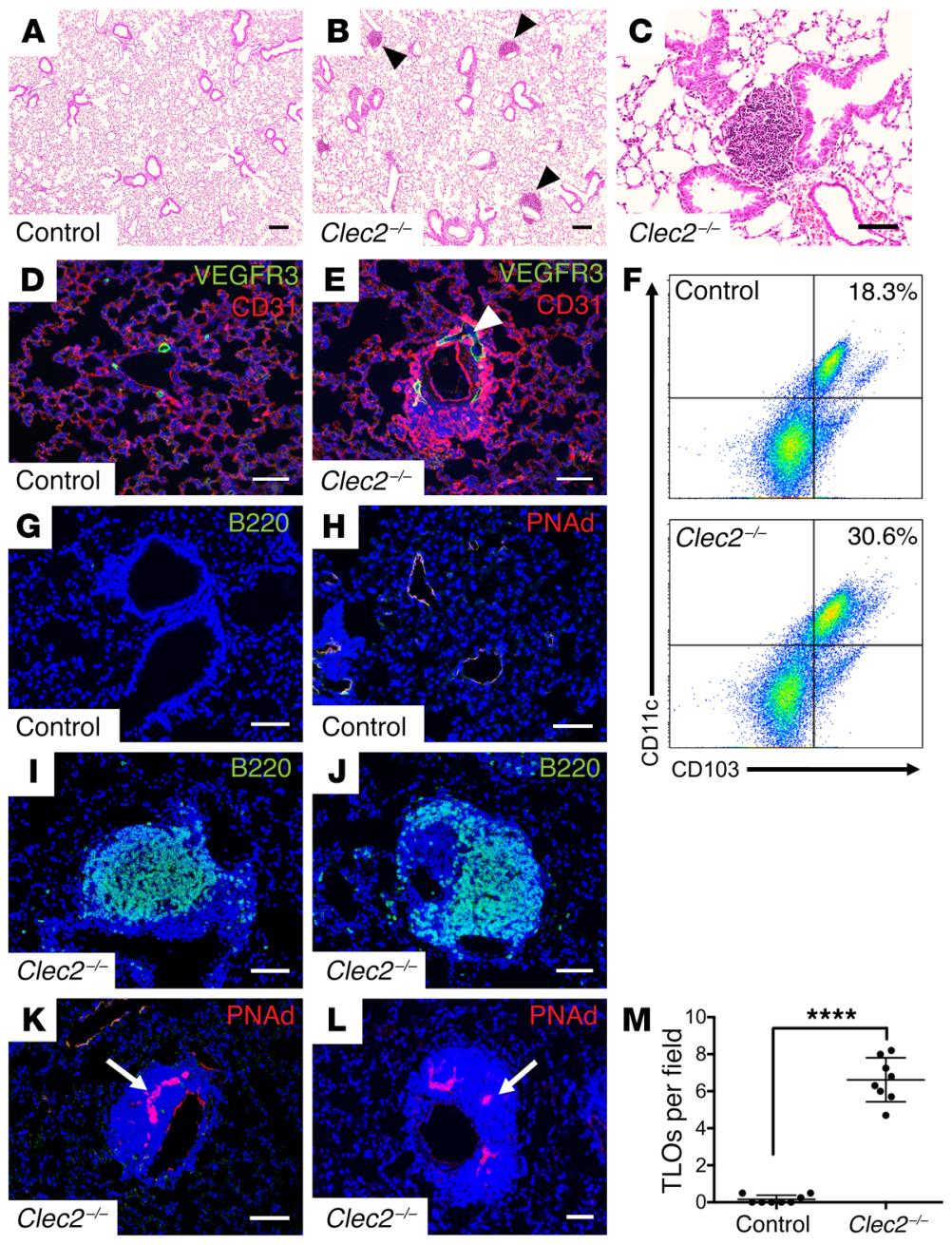

Figure 3. Loss of CLEC2 is associated with abnormal TLO formation in the lung parenchyma. (A-C) H\&E staining of lung tissue from 4- to 6-week-old $\mathrm{Clec}^{-1-}$ and control mice. Arrowheads indicate inflammatory infiltrates. (D and E) Staining for pulmonary lymphatic vessels (VEGFR3, green) and capillaries (PECAM, red). Arrowhead indicates dilated pulmonary lymphatic vessels in the lung of a $\mathrm{Clec}^{-1-}$ mouse. (F) Flow cytometric analysis of $\mathrm{CD}_{103}{ }^{+} \mathrm{CD}_{11 c^{+}}$cells in lung tissue from 8- to 12-week-old control and $\mathrm{Clec}^{-/-}$mice. Samples were gated for $\mathrm{CD} 45^{+}$leukocytes. (G-L) Immunohistochemical analysis of B cells (B220, green) and HEVs (PNAd, red, arrows) from $\mathrm{Clec}^{-/-}$and control mice. (M) Quantification of TLOs in the lungs of 8- to 12-week-old $\mathrm{Clec}^{-1-}$ mice compared with control littermates per $\times 4(1.1 \times 1.3 \mathrm{~mm})$ microscopic field. Data representative of at least 5 mice in each group. Scale bars: 100 $\mu \mathrm{m}(\mathbf{A}$ and $\mathbf{B})$ and $25 \mu \mathrm{m}(\mathbf{C}-\mathbf{L})$. All values represent the mean \pm SEM. ${ }^{* * *} P<0.0001$, by Student's $t$ test.

$\mathrm{Clec}^{2-}$ animals and iClec2-KO animals share a common lymph-venous pathology, these studies suggest that the findings in the $\mathrm{Clec}^{--}$animals described above arose because of loss of lymphatic flow. However, since lymphatic valve development requires lymph flow, the observation that pulmonary lymphatics in $\mathrm{Clec}^{--}$mice have valves (Figure 2, B and C) indicates some residual lymph flow in these animals (29).

CLEC2 deficiency results in the formation of tertiary lymphoid organs in the lungs. In addition to drainage of extracellular fluid, lymphatics serve as conduits for immune cell migration from the lungs to draining LNs, where responses to infection and inflammation are coordinated $(34,35)$. By 4 weeks after birth, we observed peribronchovascular infiltrates in the lungs of $\mathrm{Clec}^{-1-}$ mice but not in the lungs of control littermates (Figure

remodeling characterized by increased and aberrant SMC recruitment (29). Consistent with prior observations in the mesentery, we observed increased SMC coverage on pulmonary lymphatics in 4- to 8-week-old $\mathrm{Clec}^{-/-}$mice but not on pulmonary lymphatics in control animals (Figure 2, G-I).

Since constitutive CLEC2 deficiency may lead to altered lymphatics due to loss of flow-dependent lymphatic vessel remodeling such as valve formation, as well as impaired LN development (29, 30 ), we next assessed pulmonary lymphatic function in mature mice (aged 8-10 weeks), in which a conditional Clec2 allele (31) was inducibly deleted following tamoxifen administration using a Rosa26Cre ${ }^{E R T 2}$ driver (Clec $2^{f / f l}$ Rosa26Cre $e^{E R T 2}$, hereafter referred to as iClec2-KO mice). As observed in Clec2 $2^{--}$animals, iClec2KO mice exhibit reduced lymph flow due to loss of lymph-venous hemostasis and retrograde filling of the lymphatic network with blood that impairs forward lymph flow (13). To measure pulmonary lymphatic flow, Alexa Fluor 568-labeled dextran was administered intratracheally and subsequently detected in the mediastinal lymph nodes (mLNs) that drain the pulmonary lymphatics (32, 33). Drainage of Alexa Fluor 568-dextran to mLNs was significantly decreased in iClec2-KO animals, as assessed by both visual examination of the LNs using fluorescence microscopy (Figure 2, J and $\mathrm{K}$ ) and by quantification of $\mathrm{LN}$ fluorescence (Figure 2L), consistent with impaired pulmonary lymphatic function. Since
$3, \mathrm{~A}-\mathrm{C})$. These infiltrates arose in close physical proximity to the dilated pulmonary lymphatic vessels in $\mathrm{Clec}^{2-/}$ mouse lungs (Figure 3, D and E) and were not observed in littermate control lungs at any time point (Figure 3M). Flow cytometric and immunohistochemical analyses of lung tissue from 8- to 12-week-old $\mathrm{Clec}^{-1-}$ mice revealed a marked increase in the number of $\mathrm{CD} 11 \mathrm{C}^{+} \mathrm{CD} 103^{+}$ DCs as well as $\mathrm{B}^{2} 2 \mathrm{O}^{+} \mathrm{B}$ cells compared with control lungs (Figure 3, F, G, I, and J). We also observed an increase in $\mathrm{CD}^{+} \mathrm{T}$ cells in $\mathrm{Clec}^{--}$lungs (Supplemental Figure 3). The cellular organization and location of these inflammatory infiltrates was typical of TLOs. TLOs, also known as iBALT, form in response to chronic inflammation $(10,36)$. TLOs resemble secondary lymphoid tissue in their organization, and a hallmark of TLOs is the presence of high endothelial venules (HEVs), which are specialized blood vessels found exclusively in lymphoid tissue that enable homing of leukocytes to that site from the blood $(9,10)$. Consistent with their identification as TLOs, the inflammatory infiltrates in 8- to 12-week-old Clec2 $2^{--}$mouse lungs were organized around cells expressing peripheral lymph node addressin (PNAd) (Figure 3, K and L), a specific marker of HEV endothelium (37), which was not found in control lungs (Figure 3H). Importantly, we did not detect TLO formation in other tissues in $\mathrm{Clec}^{--}$mice at baseline, even in flow to an extent equal to or greater than that observed in the lungs organs such as the gut that experience a loss of forward lymphatic 

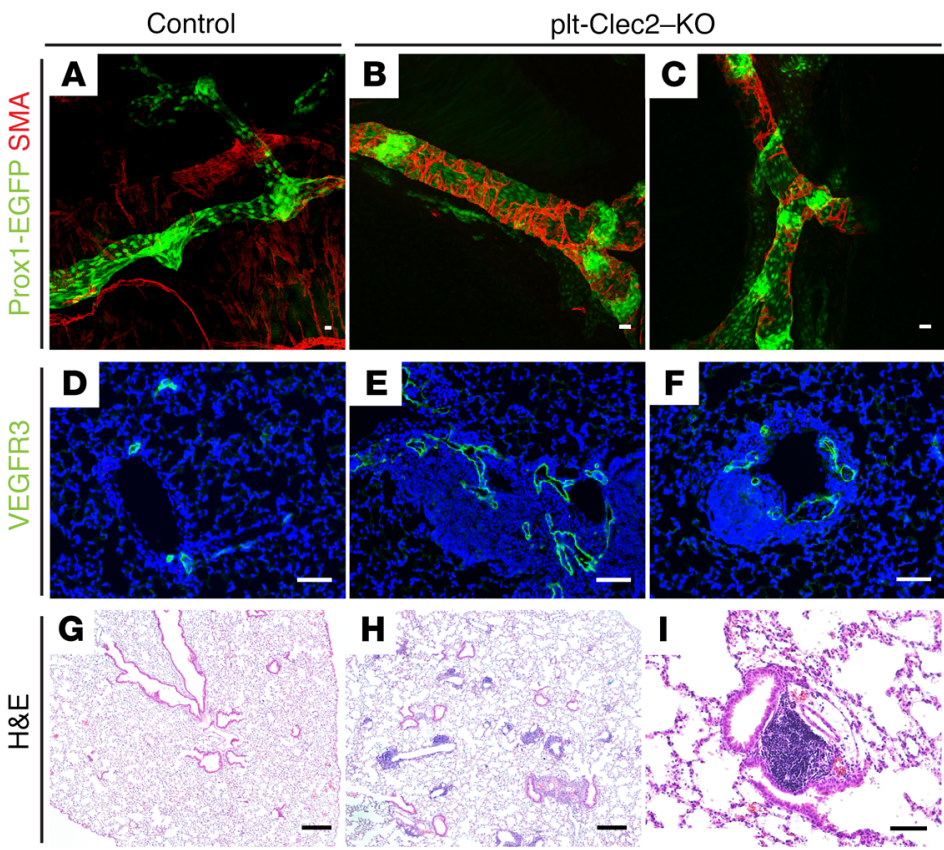

plt-Clec2-KO
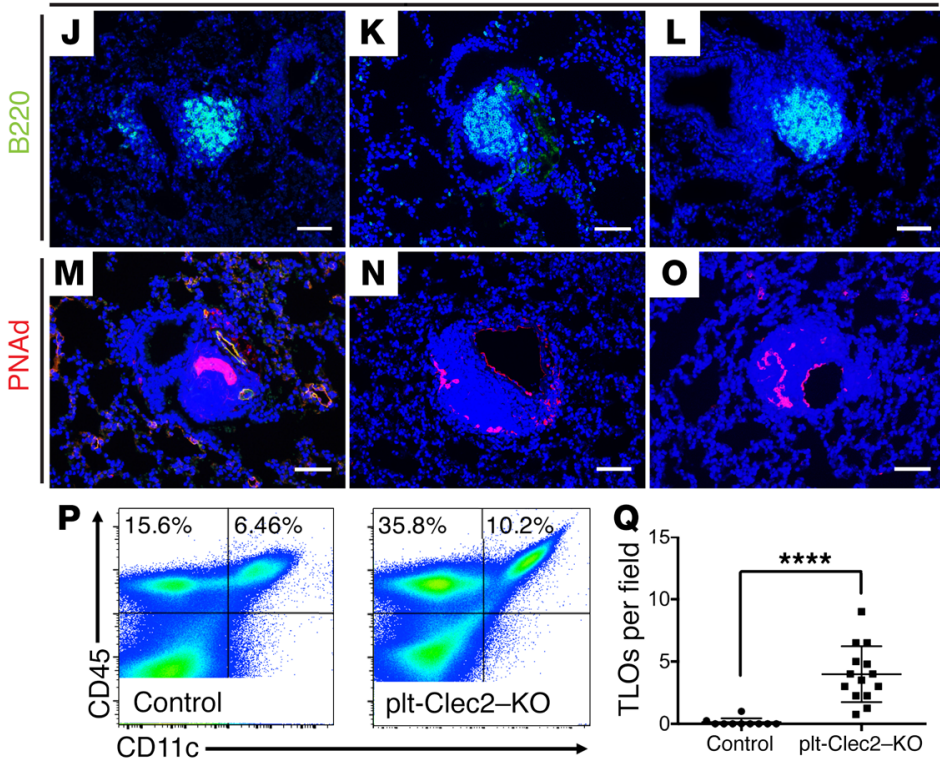

Figure 4. Platelet-specific loss of CLEC2 results in abnormal pulmonary lymphatic morphology and TLO formation in the lung parenchyma. (A-C) Whole-mount SMA staining (red) of pulmonary lymphatic vessels (Prox1-EGFP, green) from 6- to 8-weekold plt-Clec2-KO and control mice. (D-F) Immunohistochemical analysis of lung tissue sections stained for VEGFR3 (green) shows lymphatic vessels of 6 - to 8-week-old plt-Clec2-KO and control mice. (G-I) H\&E-stained images of lung tissue from 8- to 12-week-old plt-Clec2-KO and control mice. (J-0) Immunohistochemical analysis of B cells (B220, green) and HEVs (PNAd, red) from plt-Clec2-KO and control mice. (P) Flow cytometric analysis of $\mathrm{CD}^{2} 5^{+} \mathrm{CD} 11 \mathrm{c}^{+}$cells in lung tissue from control and plt-Clec2-KO mice. (Q) Quantification of TLOs in the lungs of 8- to 12-week-old plt-Clec2-KO mice compared with control littermates per $\times 4$ (1.1 $\times 1.3 \mathrm{~mm}$ ) microscopic field. Data are representative of at least 5 mice in each group. Scale bars: $25 \mu \mathrm{m}(\mathbf{A}-\mathbf{F}$ and $\mathbf{I}-\mathbf{0})$ and $100 \mu \mathrm{m}$ (G and $\mathbf{H})$. All values represent the mean $\pm \mathrm{SEM}$. ${ }^{* * * *} P<0.0001$, by Student's $t$ test.

nary lymphatics in plt-Clec2-KO animals were dilated and had abnormal SMC coverage (Figure 4, A-F). pltClec2-KO mice also developed TLOs in the lungs, with histologic features that were indistinguishable from those observed in $\mathrm{Clec}^{--}$mice, including proximity to lymphatic vessels, large numbers of $\mathrm{B}$ cells, and the presence of PNAd ${ }^{+} \mathrm{HEVs}$ (Figure 4, G-O). Flow cytometric analysis of lung tissue from plt-Clec2-KO mice revealed an increase in $\mathrm{CD} 11 \mathrm{c}^{+}$leukocytes compared with those in control lungs (Figure 4P), similar to what we observed in $\mathrm{Clec}_{2}--$ mice. While TLOs were exceedingly rare in control lungs (Figure 4 and Supplemental Figure 5), we found them throughout the lungs of 8- to 12-week-old plt-Clec2-KO mice (Figure 4Q). These findings support the conclusion that impaired lymph flow is sufficient to cause TLO formation in the lungs.

CLEC2-deficient mice exhibit defects in the drainage of fluid and cells from the lungs. We have previously shown that lymphatic drainage of interstitial fluid is required to increase lung compliance prior to birth, thereby enabling successful neonatal lung inflation (2). In contrast, a classic model originally proposed by Ernest Starling maintains that in the mature lung, fluid balance is maintained by opposing osmotic and hydrostatic pres-

(29) (Supplemental Figure 4). Whether TLO formation occurs in other organs in $\mathrm{Clec}^{2-}$ mice in response to infection or inflammation is not known.

The observation that TLOs form in proximity to dilated lymphatic vessels in CLEC2-deficient animals suggested that TLOs arise as a result of impaired lymphatic function. However, since CLEC2 is also expressed on DCs and is required for DC migration and LN development $(30,38)$, it is possible that TLO formation reflects a requirement for CLEC2 in DCs or other leukocytes rather than a loss of forward lymph flow. To address this possibility, we examined the lungs of mice with platelet-specific loss of CLEC2 ( $\mathrm{Clec} 2^{2 t /}-\mathrm{PF} 4 \mathrm{Cre}$, hereafter referred to as plt-Clec2-KO mice), which exhibit impaired lymphatic flow due to loss of lympho-venous hemostasis but retain DC expression of CLEC2 and have normal LN development (29). As in Clec2 $2^{-/}$mice, pulmo- sures in blood capillaries and surrounding tissue $(39,40)$. In this model, forces that would move fluid from the blood into the interstitium (i.e., positive capillary blood pressure, positive osmotic pressure of interstitial fluid, and negative interstitial pressure in the lung) are precisely balanced by those that would move fluid from the interstitium into the blood (i.e., positive blood osmotic pressure), leaving little role for lymphatic function. Indeed, some large animal physiologic studies have revealed a relatively minor role for pulmonary lymphatics in clearing lung fluid, even in conditions of pulmonary edema $(3,41,42)$, although invasive lymphatic flow measurements have documented increased lymphatic flow rates in settings of chronic edema $(43,44)$. Thus, whether and to what extent lung lymphatics are required to prevent pulmonary edema in the mature lung has not been established. We did not find any increase in pulmonary edema in plt-Clec2-KO mice at 
A

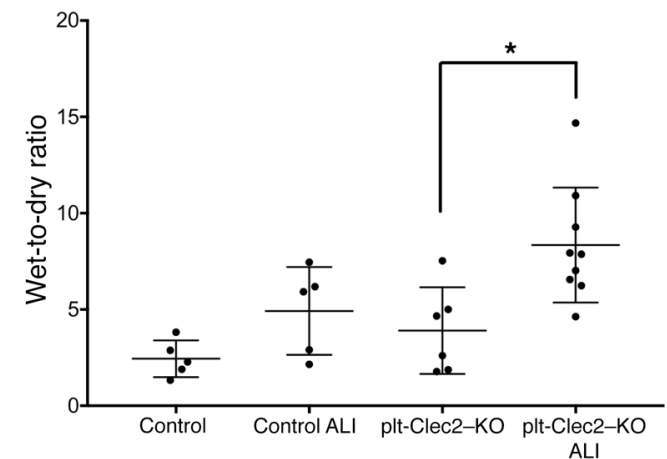

B
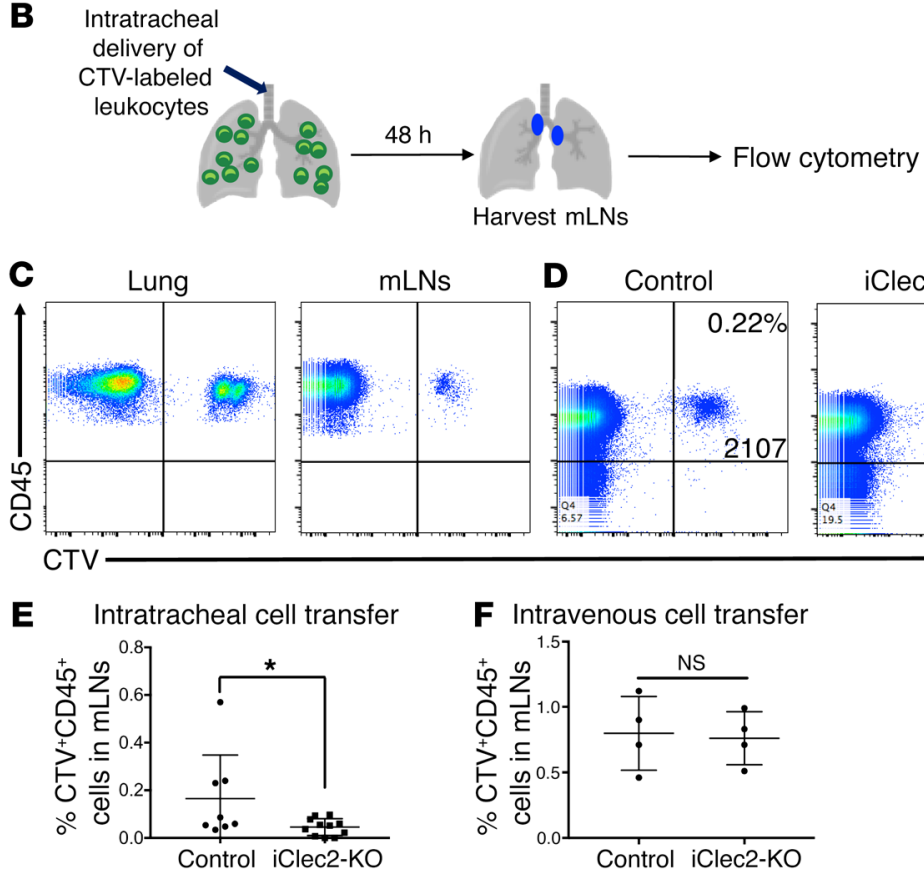

D Control
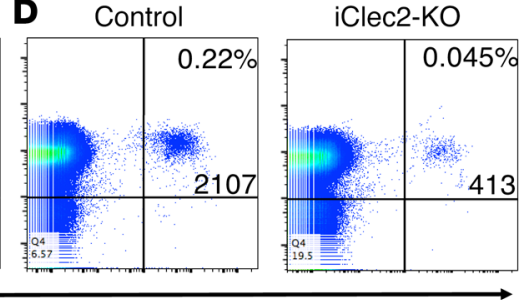

Figure 5. Loss of CLEC2 results in impaired drainage of fluid and cells from the lungs. (A) Wet-to-dry ratios for control and plt-Clec2-KO lungs at baseline and in response to acid-induced lung injury (ALI). (B) Schematic of cell-tracing experiment for assessment of lymphatic leukocyte trafficking from the lungs to draining LNs. Intratracheal administration of CTV-labeled leukocytes was followed by harvesting of mLNs for flow cytometric analysis. (C) Identification of $\mathrm{CD} 45^{+} \mathrm{CTV}^{+}$leukocytes in the lungs and mLNs of WT mice 48 hours after intratracheal administration. (D) Flow cytometric analysis of $\mathrm{CD}_{4}{ }^{+} \mathrm{CTV}^{+}$leukocytes in $\mathrm{mLNs}$ from iClec2-KO and control mice, 48 hours after intratracheal administration. (E) Quantification of CTV+ leukocytes in $\mathrm{mLNs}$ from control and iClec2-KO mice after intratracheal administration, as a percentage of total $\mathrm{CD} 45^{+}$leukocytes. (F) Quantification of $\mathrm{CTV}^{+}$leukocytes in $\mathrm{mLNs}$ from control and iClec2KO mice after intravenous administration, as a percentage of total $\mathrm{CD} 45^{+}$leukocytes. Data are representative of at least 4 mice in each group. All values represent the mean \pm SEM. ${ }^{*} P<0.05$, by Student's $t$ test. baseline using a wet-to-dry ratio (Figure 5A), consistent with the lack of histologic changes indicative of edema in the lungs of pltClec2-KO mice (Figure 4, $\mathrm{H}$ and I, and data not shown). In contrast, plt-Clec2-KO but not control mice exhibited significant pulmonary edema after acid-induced lung injury (Figure 5A).

The accumulation of leukocytes and TLO formation in mice with reduced lymphatic function due to CLEC2 deficiency suggested that a primary function of pulmonary lymphatics is to transport immune cells from the lungs to the draining mLNs. Migration of leukocytes from the lungs to LNs after infection is essential for the adaptive immune response $(34,45)$. However, the fact that TLO formation was observed in the lungs of CLEC2deficient mice in the absence of infection suggested that lymphatic function may be required for leukocyte trafficking from the lung, even under healthy conditions. To measure trafficking, leukocytes labeled with CellTrace Violet (CTV) were administered to mice intratracheally and subsequently detected by flow cytometry in the lungs and draining mLNs of control and iClec2-KO mice at least 4 weeks after tamoxifen treatment (45) (Figure 5, B and C), a time point previously found to be associated with impaired pulmonary lymphatic flow (Figure 2, J-L). Migration of CTV-labeled leukocytes from the airways to $\mathrm{mLNs}$ via lymphatics was significantly decreased in iClec2-KO mice (Figure 5, D and E). Consistent with this finding, we detected increased numbers of leukocytes following bronchoalveolar lavage (BAL) of plt-Clec2-KO mice compared with control animals (Supplemental Figure 6). In contrast, we found that movement of leukocytes to mLNs following intravenous administration was not affected in iClec2-KO mice (Figure $5 \mathrm{~F})$. These findings suggest that TLO formation in CLEC2-deficient mice results from the accumulation of immune cells in the lungs due to loss of pulmonary lymphatic function that is required for immune cell egress under basal conditions.

Lung-specific lymphatic ablation results in rapid TLO formation in the lung parenchyma. The studies using CLEC2-deficient animals described above suggested that loss of lymphatic vascular function results in TLO formation specifically in the lungs. However, because lymphatic flow is systemically impaired in CLEC2deficient animals, it is possible that TLO formation resulted from global changes in immune cell activation and/or migration rather than a lung-specific requirement for lymph flow. Furthermore, we could not rule out the possibility that TLOs formed in the lungs of CLEC2-deficient mice as a result of contact with blood rather than as a consequence of impaired lymphatic flow. To more precisely test the role of lymphatic vascular function in the lungs and to rule out any unexpected secondary effects from blood-filled lymphatics in CLEC2-deficient models, we sought to generate mice in which 
A
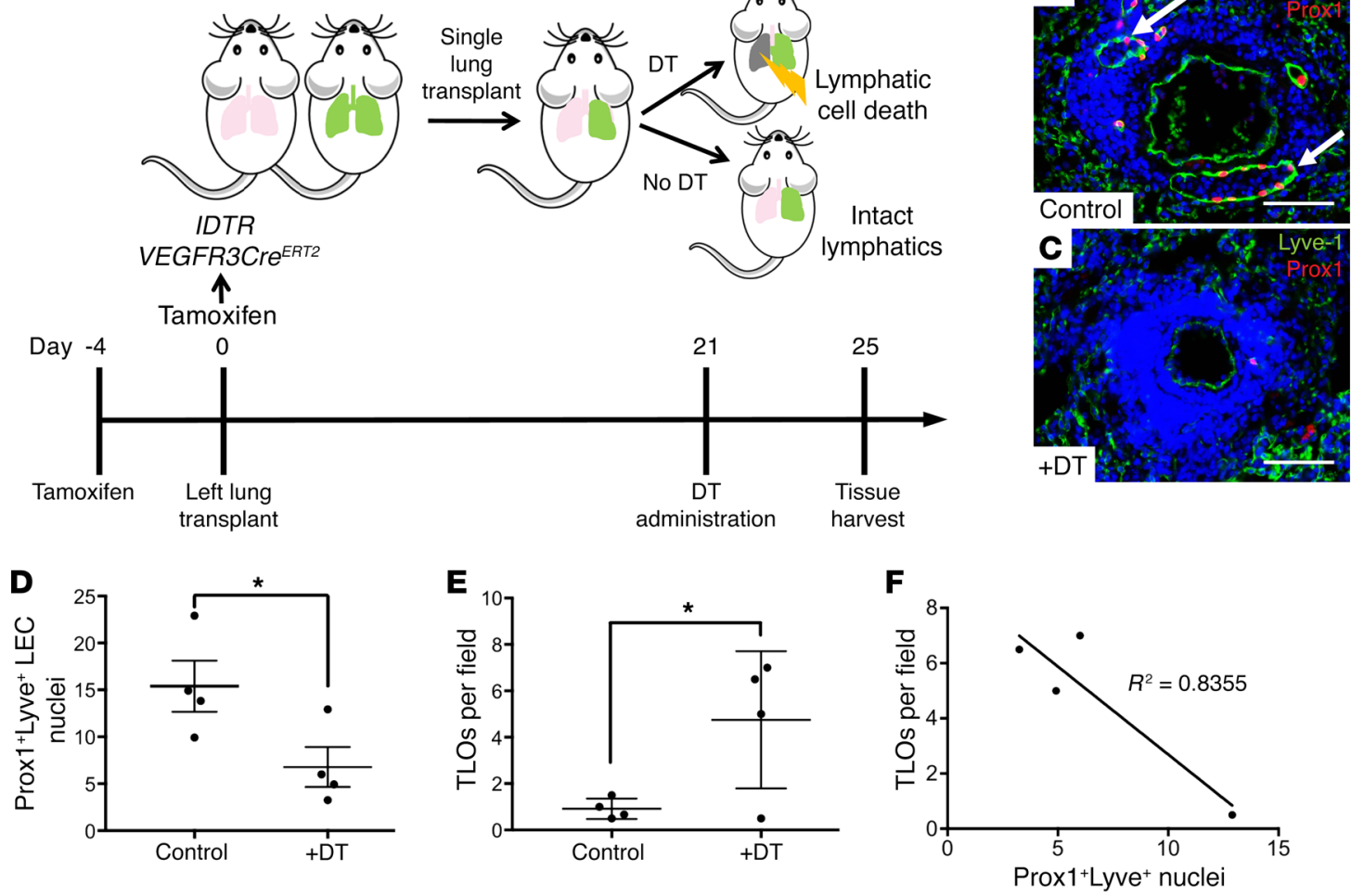

Figure 6. Lung-specific LEC ablation leads to TLO formation. (A) Schematic of the experimental approach used for lung-specific deletion of pulmonary lymphatics. iDTR expression was induced in LECs using LEC-specific Cre (VEGFR3Cre ${ }^{E R T 2}$ ). Lungs from iDTR VEGFR3Cre ${ }^{E R T 2}$ mice were used as donors for single lung transplantation into littermate recipients, and administration of DT to transplanted mice led to LEC death specifically in the transplanted lung, whereas control transplanted lungs had intact lymphatics. Timeline shows lung-specific lymphatic ablation in the lung transplants. (B and C) Immunohistochemical analysis of Prox $1^{+}$Lyve1 $1^{+}$LEC nuclei in control lungs (B, arrows) and lungs with DT-mediated lymphatic ablation (C). (D) Quantification of Prox $1^{+}$

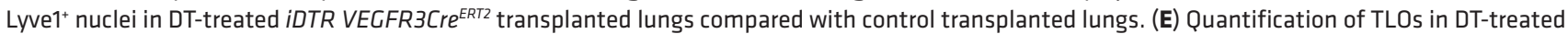
IDTR VEGFR3Cre ${ }^{E R T 2}$ transplanted lungs compared with control transplants per $\times 4(1.1 \times 1.3 \mathrm{~mm})$ microscopic field. (F) Correlation of TLOs to the number of Prox1+Lyve ${ }^{+}$LEC nuclei in DT-treated iDTR VEGFR3Cre ${ }^{E R T 2}$ transplanted lungs. Data are representative of 4 mice in each group. Scale bars: $25 \mu \mathrm{m}$. All values represent the mean \pm SEM. ${ }^{*} P<0.05$, by Student's $t$ test.

lymphatic function could be specifically ablated in the adult lung. To accomplish this, we used mice expressing both Cre-inducible diphtheria toxin receptor (iDTR) (46) and tamoxifen-inducible Cre recombinase driven by the lymphatic-specific VEGFR3 promoter (47) (iDTR VEGFR3Cre ${ }^{E R T 2}$ ) as donors for lung transplantation (Figure 6A). VEGFR3 expression is restricted to LECs in the mature lung, and lineage tracing confirmed that the VEGFR3Cre ${ }^{E R T 2}$ transgene was active specifically in those cells (Supplemental Figure 7). Tamoxifen administration to iDTR VEGFR3CreERT2 mice induced the expression of iDTR in LECs of the donor lungs, rendering all LECs in the lung susceptible to cell death following exposure to diphtheria toxin A (DT). The left lungs from donor iDTR VEGFR3$\mathrm{Cre}^{E R T 2}$ mice were transplanted into control littermates. Although lymphatic vessels were not surgically reconnected after lung transplantation, studies in mice and large animals have demonstrated that lymphatic drainage is reestablished 7-21 days after transplantation $(33,48,49)$. We administered DT 21 days after transplantation of the iDTR VEGFR3Cre ${ }^{\text {ERT2 }}$ lung to ensure that lymphatic function was first reestablished in the transplanted lungs (Figure $6 \mathrm{~A})$. Rejection of the transplanted lung was prevented by backcrossing with mice on a C57Bl/6 background and by the strict use of littermates for donor and recipient animals.
Immunostaining of the $i D T R$ VEGFR3Cre ${ }^{E R T 2}$ lung isografts for the lymphatic endothelial markers Lyve1 and Prox1 five days after onset of DT administration revealed a greater than 50\% loss of LECs compared with control transplanted lungs (Figure $6, \mathrm{~B}-\mathrm{D})$, a finding predicted to impair lymphatic function. We did not observe a significant amount of blood in the lymphatics of control transplanted lungs or in the remaining lymphatics in transplants with DT-mediated lymphatic deletion (Supplemental Table 2), confirming that this model does not result in retrograde blood flow into the lymphatic vessels, as seen in CLEC2-deficient mice. Of significant note, we observed abundant TLO formation, marked by the presence of large numbers of $\mathrm{B}^{2} 2 \mathrm{O}^{+}$cells and PNAd ${ }^{+} \mathrm{HEVs}$, in iDTR VEGFR $3 \mathrm{Cr} \mathrm{ERT2}^{\text {ET2 }}$ transplanted lungs that were treated with DT, but not in control transplanted lungs with intact lymphatics (Figure 6E). We observed a strong correlation between the extent of LEC loss and the formation of TLOs, as greater numbers of TLOs were detected in transplanted lungs that showed greater deletion of LECs (Figure 6F). The TLOs that formed after lymphatic deletion closely resembled those in the lungs of plt-Clec2-KO mice in terms of their location, appearance, and cellular composition (Figure 7, A-P). The rapidity with which TLOs formed after lymphatic deletion in this model (with- 

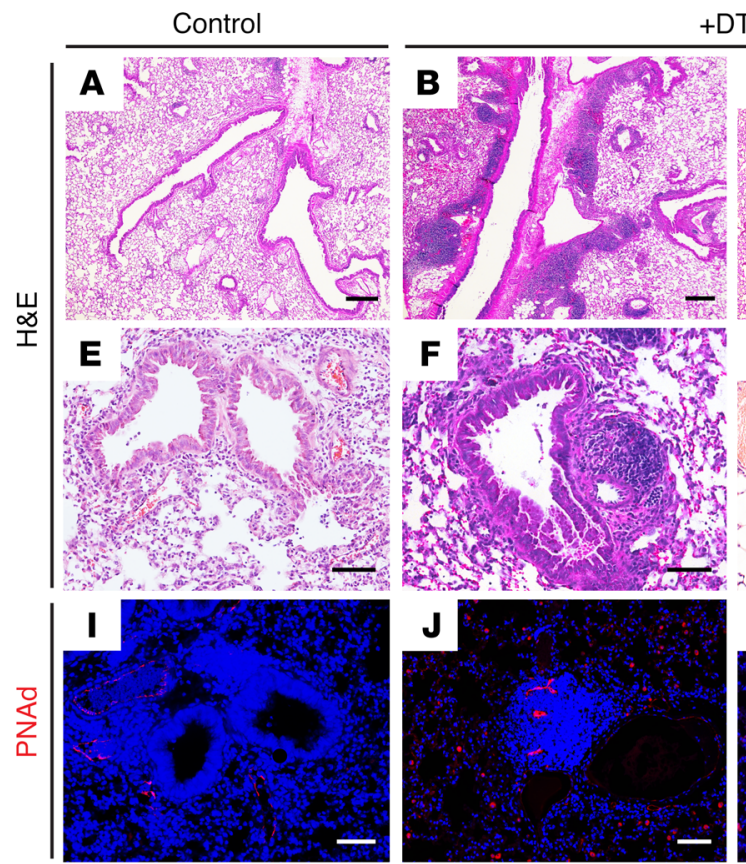

$+\mathrm{DT}$
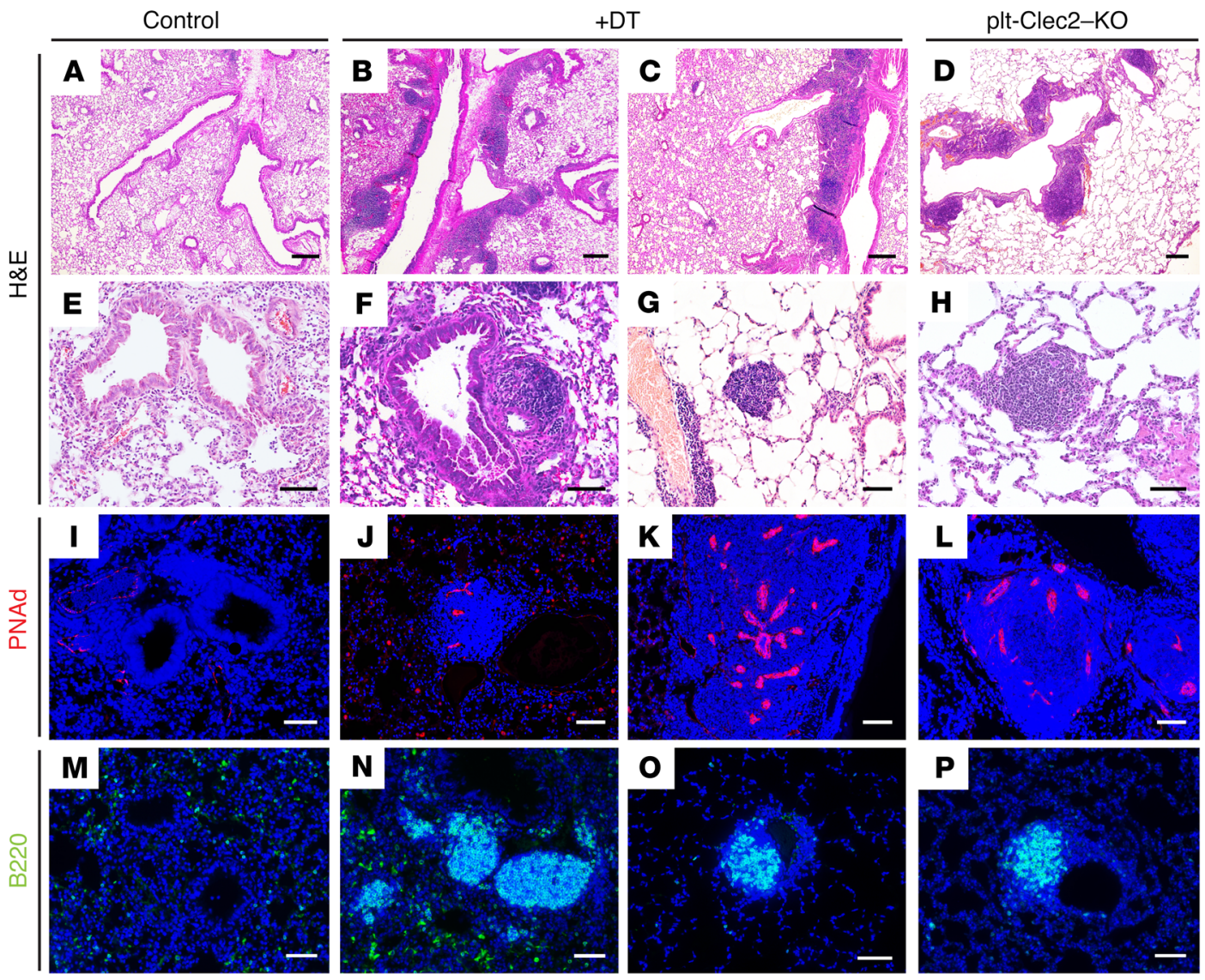

Figure 7. TLOs after lung lymphatic ablation resemble those in plt-Clec2-KO mice. (A-H) H\&E-stained images of lung tissue from control transplanted lungs, DT-treated IDTR VECFR3Cre ERT2 $^{2}$ transplanted lungs with lymphatic ablation, and lungs from plt-Clec2-KO mice. (I-P) Immunohistochemical analysis

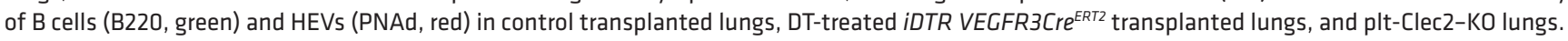
Data are representative of 4 mice in each group. Scale bars: $100 \mu \mathrm{m}$ (A-D) and $25 \mu \mathrm{m}$ (E-P).

in 5 days) compared with those in CLEC2-deficient mice (at least 4 weeks) was notable. This likely reflects the effects of acute and severe disruption of lymphatic flow in this model, as opposed to the more mild and chronic impairment of lymphatic flow conferred by loss of CLEC2. These results provide a second line of genetic evidence to support the conclusion that loss of lymphatic function in the lung results in TLO formation.

Constitutive loss of CLEC2 results in an emphysematous lung phenotype. TLO formation is commonly seen in chronic lung inflammation and is associated with diverse lung diseases in humans $(10,36)$, but whether TLO formation is connected to impaired lymphatic flow, and whether TLOs are a cause or a consequence of lung disease, remains unclear $(50,51)$. The primary formation of TLOs in mice with impaired lymphatic flow led us to ask whether lymphatic impairment might result in lung injury and pathology. Although TLOs formed as early as P35 in plt-Clec2-KO mice, we did not observe any significant lung parenchymal changes at this time point (Supplemental Figure 8). However, 6- to 8-month-old plt-Clec2-KO mice had marked alveolar enlargement similar to that observed in the human lung disease emphysema (Figure $8, \mathrm{~A}-\mathrm{C}$ ). This was not due to a primary defect in alveolarization in these mice, as there was no appreciable alveolar enlargement prior to P36 (Supplemental Figure 8), a time point by which alveolar development is nearly complete (52). The emphysematous alveolar phenotype in plt-Clec2-KO mice was associated with hypoxia, an indicator of significant impairment of lung function (Figure 8D). A hallmark of human emphysema is the breakdown of elastin in the alveolar walls due to secretion of degrading proteases by macrophages and neutrophils (53, 54). Immunostaining revealed reduced elastin in lungs from pltClec2-KO mice compared with those from control littermates, particularly in areas where the emphysema phenotype was most pronounced (Figure 8, E and F). Severe emphysema in humans is associated with the generation of elastin fragments, which are considered a marker of active disease (55) and may drive disease progression (56-58). We detected a significant increase in a $25-\mathrm{kDa}$ elastin fragment and a nonsignificant increase in a $45-\mathrm{kDa}$ elastin fragment in the lungs of 6- to 8-month-old plt-Clec2-KO mice compared with lungs of control animals (Figure 8, G and $\mathrm{H}$, and Supplemental Figure 9). Expression of the elastin-degrading matrix mettaloproteinase-12 (MMP-12) is required for the development of cigarette smoke-induced emphysema in mice and is upregulated in humans with this disease $(53,59,60)$. Increased MMP-12 was detected in the lungs of mice with loss of CLEC 2 by both quantitative PCR (qPCR) and immunohistochemistry (Figure $8, \mathrm{I}-\mathrm{K})$. Since the only described effect of CLEC2 deficiency 

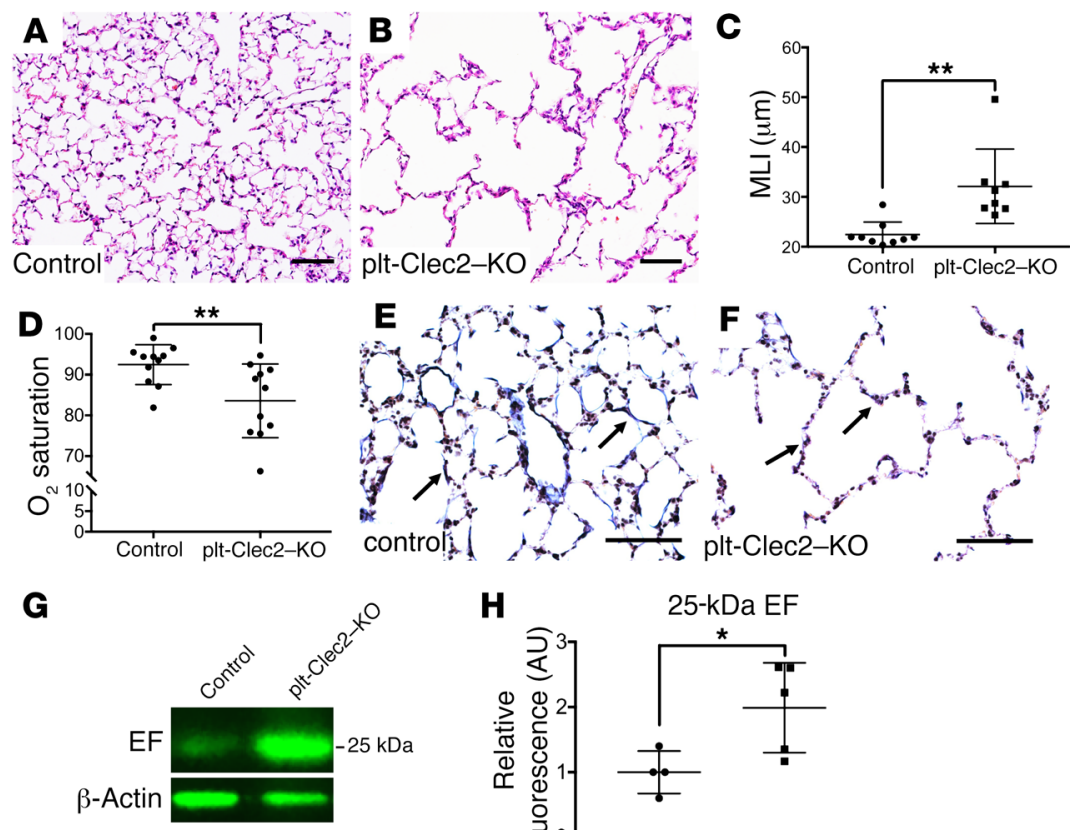

$\beta$-Actin
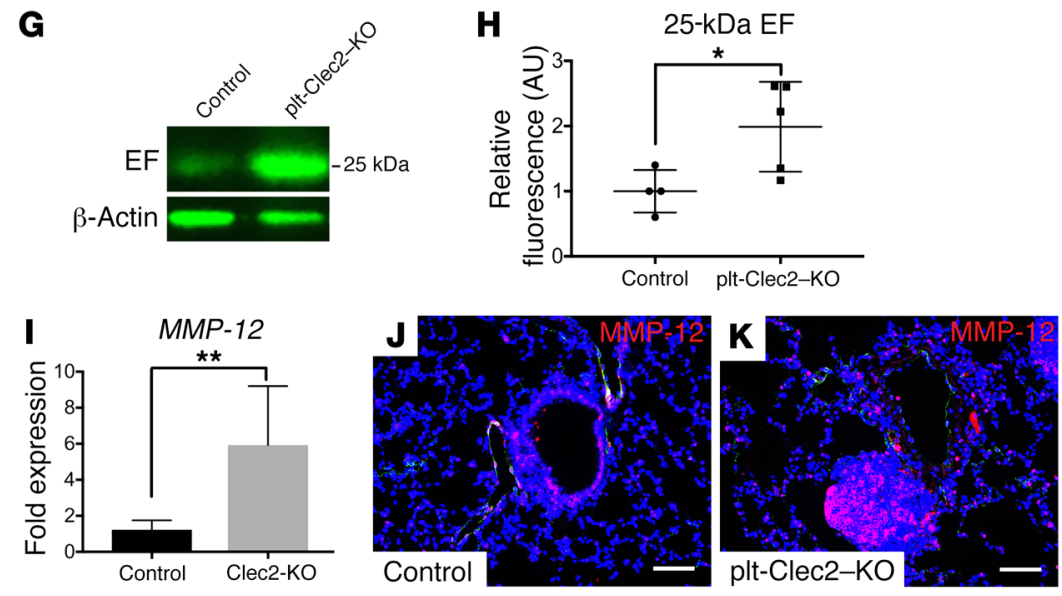

Figure 8. CLEC2 deficiency results in reduced lung function associated with emphysematous changes. (A and $B$ ) H\&E-stained images of lung tissue from 6- to 8-monthold plt-Clec2-KO and control mice. (C) Quantification of alveolar enlargement in 6- to 8-month-old plt-Clec2-KO and control mice by mean linear intercept (MLI). (D) Oxygen saturation in 6- to 8-month-old plt-Clec2-KO and control mice. (E and F) Van Gieson staining for elastin (black, arrows) in lung tissue from control and plt-Clec2KO mice. (G) Western blot of whole lung tissue from 6 - to 8-month-old plt-Clec2-KO and control mice for detection of the $25-\mathrm{kDa}$ elastin fragment (EF). (H) Quantification of the $25-\mathrm{kDa}$ elastin fragment in Western blots by fluorescence intensity $(\mathrm{AU})$. Comparisons were made between matched littermates. (I) qPCR for MMP-12 expression in lung tissue from plt-Clec2-KO and control mice. Comparisons were made between matched littermates. (J and $\mathbf{K}$ ) Immunohistochemical analysis of MMP-12 (red) in lung tissue from plt-Clec2-KO and control mice. Data are representative of at least 4 mice in each group. Scale bars: $25 \mu \mathrm{m}$. All values represent the mean \pm SEM. ${ }^{*} P<0.05$ and ${ }^{* *} P<0.01$, by Student's $t$ test. on the lungs is impaired lymphatic flow, these results suggest that chronic loss of lymphatic function is sufficient to confer a lung phenotype with molecular and cellular features of emphysema.

\section{Discussion}

Organ-specific roles have recently emerged as a central mechanism by which the vascular system functions in both healthy and diseased states (61), but designing studies to identify these roles in the lymphatic system is challenging, given the lack of organspecific lymphatic markers and tools to target the lymphatic vasculature of a particular organ. The present studies identify unique anatomic and functional features of pulmonary lymphatic vessels. The finding that lymphatic vessels lack SMC coverage is unique to the lungs and suggests that pulmonary lymph flow is maintained by other mechanisms, e.g., respiratory movements and changes in thoracic pressure. The inability of lung collecting lymphatics to independently pump lymph may make these vessels uniquely susceptible to blockage and dysfunction. Consistent with this idea, we observed that, following global impairment of lymphatic function, spontaneous formation of TLOs occurred specifically in the lungs. We propose that these unique anatomical and functional features are connected and that they may link changes in lymphatic vessel function to the pathogenesis of common lung diseases.

In the present study, we used 2 distinct approaches to examine the loss of pulmonary lymphatic function in the mouse: genetic deletion of CLEC2, a platelet receptor required to maintain forward pulmonary lymph flow, and the transplantation of lungs genetically engineered to allow inducible ablation of LECs. The findings from these 2 models are highly concordant and demonstrate a lung-specific role for lymphatic vessels in preventing a chronic inflammatory state characterized by the formation of TLOs. We also found that chronically impaired lymphatic flow due to loss of CLEC2 resulted in alveolar enlargement and hypoxia, with features of lung injury that resembled emphysema. These findings establish an organ-specific role for lymphatic vessels in the lung and suggest that impairment of lung lymphatic function may participate in the pathogenesis of lung diseases such as emphysema that are associated with TLO formation.

An established, and often considered universal, role of lymphatic vessels is to take up interstitial fluid and proteins that leak from the blood vasculature, thereby preventing tissue edema. Consistent with this role, a hallmark sign of generalized loss of lymphatic function is tissue edema (62), and lymphatic function is required for fluid drainage in the developing lung to enable neonatal lung inflation (2). Although large animal studies using short-term catheter-based approaches have demonstrated that lymphatic flow in the lung may be dynamic $(21,22)$, pulmonary edema can also be considered a consequence of imbalanced Starling forces in the alveolar blood capillaries rather than loss of lung lymphatic function (63). Our studies of CLEC2-deficient lungs provide genetic insight into the long-term role of lymphatic vessels in the management of fluid homeostasis in the mature lung. Lung wet-to-dry ratios were not elevated in CLEC2-deficient lungs at baseline. Though this is an insensitive measure of pul- 
monary edema, the absence of histologically apparent edema in the lungs of CLEC2-deficient mice suggests that any pulmonary edema that results from impaired lymphatic drainage is unlikely to be severe in the otherwise healthy lung. However, we did observe an increased wet-to-dry ratio in response to lung injury in CLEC2deficient mice, indicating that lymphatic dysfunction contributes to pulmonary edema in this setting and establishing a role for pulmonary lymphatics in lung fluid management. Future studies addressing pulmonary lymphatic function in other pathologic settings are likely to shed additional light on this role.

The most prominent effect of impaired pulmonary lymphatic function observed in these studies was the formation of TLOs, a pathology detected following both loss of CLEC2 and LEC ablation in transplanted lungs. Since these in vivo models share no molecular or cellular features other than loss of lymphatic function, this phenotype is highly likely to be a specific consequence of lymphatic impairment. This conclusion is also supported by the fact that TLOs were observed adjacent to collecting lymphatic vessels. Moreover, although CLEC2-deficient mice experience loss of mesenteric and intestinal lymphatic function that is similar in magnitude to that in the lung (29), TLOs are selectively observed in the lungs of CLEC2-deficient mice. Why does loss of lymphatic function result in TLO formation specifically in the lung? One possible explanation for this organ specificity is that pulmonary lymphatic vessels transport a larger number of immune cells than do lymphatic vessels in other organs because the lung is a barrier organ exposed to the outside environment. An alternative explanation is suggested by the unique anatomy of pulmonary lymphatic collecting vessels that completely lack SMC coverage. The lack of SMC coverage may make pulmonary collecting lymphatics more permeable than those in other organs and facilitate cellular extravasation when lymph flow is impeded (e.g., in the CLEC2KO model) or when the lymphatic endothelial barrier is disrupted (e.g., in the LEC ablation model). In either case, the fact that lymphatic impairment is sufficient to confer TLO formation in an otherwise healthy lung suggests that changes in lymphatic function may play an important role in lung pathology.

TLO formation is a common histologic feature of chronic lung inflammation that is observed in emphysema, lung transplant rejection, and pulmonary complications of rheumatoid arthritis (64-66). However, whether TLO formation is a cause or consequence of these disease processes has not been clear. In CLEC2-deficient mice, TLO formation in the lung is an early event that is followed by evidence of lung injury characterized by alveolar enlargement, breakdown of tissue elastin, and significant hypoxia - all features of cigarette smoke-induced emphysema in humans. The clear temporal sequence of these events, and the fact that platelet CLEC2 deficiency appears to affect only the lung indirectly through changes in lymph flow, suggest that TLO formation may be a causal mechanism in lung injury and that lymphatic dysfunction may underlie both TLO formation and lung disease pathogenesis. The fact that abnormal lymphatic vessels have been observed in a variety of chronic lung diseases (12) provides additional evidence for this idea. Future studies will need to address the precise role of lymphatic function in the pathogenesis of common lung diseases associated with TLO formation and how cigarette smoke exposure may directly affect lymphatic vessel function.

\section{Methods}

Animal models. Clec2-, Clec2 ${ }^{f l}$, PF4-Cre, and Prox1-EGFP BAC-transgenic mice have been previously described by our laboratory (23, 29) and were maintained on a mixed background. Rosa26-iDTR and Rosa26-iYFP mice were purchased from The Jackson Laboratory. $V E G F R 3 C r e^{E R T 2}$ mice (a gift from Sagrario Ortega, Spanish National Cancer Research Center, Madrid, Spain) have been previously described (47). For transplantation experiments, all mice were maintained on a C57Bl/6 background. For all experiments, both male and female mice were used.

Whole-mount staining. Tissue from mice carrying the Prox1EGFP transgene was fixed overnight in $4 \%$ paraformaldehyde (PFA) at $4^{\circ} \mathrm{C}$. For lungs, thick coronal sections were made using a scalpel. Other tissues were stained intact. Tissue was permeabilized in $0.1 \%$ BSA plus $0.3 \%$ Triton- $X$ in PBS. Incubation with Cy3-conjugated anti-SMA antibody (MilliporeSigma, C6198) was performed in $0.3 \%$ Triton- $\mathrm{X}$ in PBS. The tissue was imaged using a Leica TCS SP8 confocal microscope.

Dextran assay. For dextran assays, $50 \mu \mathrm{l}$ of $5 \mathrm{mg} / \mathrm{ml}$ dextran-568 (10,000 kDa MW, Thermo Fisher Scientific) was administered to anesthetized, intubated mice via endotracheal catheterization. Fifty minutes after administration, the mice were sacrificed for the harvesting of mLNs. This time point is based both on previous reports using this assay (33) and our own studies demonstrating the time course of dextran drainage to the mLNs after intratracheal delivery (Supplemental Figure 2). Lymph nodes were imaged using an Olympus SZX16 dissecting microscope. Quantification of fluorescence intensity was performed using Image J software (NIH).

Immunohistochemistry. Mice were sacrificed and tissue was perfused with PBS. Lungs were inflated with $4 \%$ PFA at a constant pressure of $25 \mathrm{~cm} \mathrm{H}_{2} \mathrm{O}$ prior to harvesting and fixation with 4\% PFA overnight. Slides from paraffin-embedded sections were $\mathrm{H} \& \mathrm{E}$ stained or immunostained with the following antibodies: B220 (Abcam, ab64100); CD3 (Abcam, ab16669); PNAd (BD Biosciences, 553863); Lyve1 (R\&D Systems, AF2125); VEGFR3 (R\&D Systems, AF743); Prox1 (Abcam, ab76696); MMP-12 (Abcam, ab128030); CD31 (HistoBiotec, DIA 310); and NG2 (MilliporeSigma, AB5320). Slides of normal human lung tissue were stained with antibodies against PDPN (D240, Abcam, ab77854). Elastin was detected using a Modified Verhoeff Van Gieson Stain Kit (MilliporeSigma).

Cell-tracing assay and flow cytometry. Splenocytes were isolated from WT mouse spleens and cultured overnight with $100 \mathrm{ng} / \mathrm{ml}$ LPS and $5 \mu \mathrm{g} / \mathrm{ml}$ phytohemagglutinin (PHA) (MilliporeSigma). The cells were then labeled with CTV (Molecular Probes) according to the manufacturer's instructions. CTV-labeled cells $\left(1 \times 10^{7}\right)$ were administered to anesthetized and intubated mice either via endotracheal catheter or intravenously. LNs or lungs were harvested 48 hours after administration of CTV-labeled cells. Single-cell suspensions were stained with the following antibodies: FITC-conjugated anti-CD45 (eBioscience, 11-04551-82); PE-Cy7-conjugated anti-CD11c (BD Biosciences, 561022); and PerCP/Cy5.5-conjugated anti-CD103 (BioLegend, 121415). Flow cytometry was performed using a BD FACSCanto and analyzed using FlowJo software.

$B A L$. Mice were sacrificed and BAL was then performed through a 20-gauge angiocatheter (BD Pharmingen), with the intratracheal instillation of $1 \mathrm{ml}$ PBS containing an anti-protease cocktail (MilliporeSigma) and $5 \mathrm{mM}$ EDTA given in $0.5-\mathrm{ml}$ increments. The total 
leukocyte cell count (cells/ml BAL fluid) was measured using a Coulter Cell and Particle Counter (Beckman Coulter).

qPCR and Western blotting. Total RNA was isolated from lung tissue using the RNeasy Kit (QIAGEN). cDNA was made using the Superscript III First-Strand Synthesis System (Invitrogen, Thermo Fisher Scientific) according to the manufacturer's instructions. qPCR analysis of gene expression was performed using the QuantStudio 6 Real-Time PCR System and SYBR Green PCR Master Mix (Applied Biosystems). Analysis of relative gene expression was performed with the comparative Ct method $(\triangle \mathrm{Ct})$, using GAPDH as the reference housekeeping gene. Each qPCR reaction was performed in triplicate. Western blotting was performed according to standard protocols and probed with anti-elastin (Abcam, ab21610) and anti- $\beta$-actin antibodies (Abcam, ab8226). Blots were imaged using a LI-COR Imaging System and quantified with Image J software.

Acute lung injury. Mice were exposed to acid-induced lung injury as previously described (67). Briefly, $30 \mu \mathrm{l}$ of $0.1 \mathrm{~N} \mathrm{HCl}$ was administered to anesthetized and intubated mice via endotracheal catheter. Anesthetized mice were ventilated for 1 hour after acid administration, at which time they were euthanized for tissue harvesting. Left lungs were weighed immediately after removal (wet weight) and again after drying in an oven at $55^{\circ} \mathrm{C}$ for 48 hours (dry weight).

Lung transplants and pulmonary lymphatic ablation. iDTR VEGFR$3 \mathrm{Cr} e^{E R T 2}$ mice were used as donors for single lung transplants into control littermates. Expression of the DTR was induced by administering tamoxifen intraperitoneally for 5 consecutive days prior to transplantation. The heart-lung block was harvested and flushed with heparin and saline, and the left lung was then isolated. The recipient mouse was anesthetized with isoflurane, intubated, and connected to a ventilator. The mouse was then placed in the supine position and an incision made in the left chest between the third and fourth ribs to expose the left pulmonary artery and vein. After a micro-serrefine clamp (FST 18055) was placed at the left hilum, a transverse incision was made on the left bronchus. A running stitch of 11-0 suture (Ethilon 2881G) was used for end-to-end anastomosis of donor-to-recipient bronchus. The donor pulmonary artery and vein were connected to the recipient pulmonary artery and vein, respectively, end to side, using the same 11-0 suture. The native left lung was removed after implantation of the donor lung. Lymphatic deletion in transplanted lungs was induced 21 days after transplantation by administration of $5 \mu \mathrm{g}$ diptheria toxin (MilliporeSigma) over 72 hours using a subcutaneous pump (Alzet).

Assessment of oxygen saturation. Mice were anesthetized using isoflurane, and pulse oximetry was performed using a MouseSTAT (Kent Scientific). For each mouse, oxygen saturation was recorded every 10 seconds for 1 minute, and these values were averaged. Mice were monitored and anesthesia was adjusted to ensure that the heart rate was maintained at more than $400 \mathrm{bpm}$ for all measurements and oxygen saturation was consistently detected.

Statistics. Quantification of TLOs in lung tissue was performed using $\times 4$ images $(1.1 \times 1.3 \mathrm{~mm})$, with at least 3 images used for each mouse. TLOs were defined as discrete leukocyte-dense accumula- tions on H\&E-stained sections that were predominantly B cells or had PNAd $^{+}$HEVs by immunohistochemical analysis of serial sections. Quantification of SMA staining on VEGFR3 ${ }^{+}$lymphatics was done on double-stained slides of lung tissue from control mice. Thirty randomly selected VEGFR $3^{+}$lymphatic vessels were imaged at $\times 20$ magnification, and each vessel was assessed for any associated SMA staining. Quantification of $\mathrm{Prox}^{+}{ }^{+} \mathrm{Lyve}^{+}{ }^{+}$endothelial cells was performed by scoring double-positive nuclei using $\times 20$ images of immunostained tissue from lung transplants. At least 10 randomly selected images were used for each mouse. Quantification of the mean linear intercept was performed as previously described (68). Briefly, the sum of the lengths of lines drawn across the images was divided by the number of intersections between alveolar walls. At least ten $\times 20$ images of lung tissue from each mouse were used for analysis. Data are expressed as the mean \pm SEM, and the numbers of samples per condition are indicated in the figure legends. Statistical significance was determined by unpaired, 2-tailed Student's $t$ test or ANOVA using GraphPad Prism software. $P$ values of less than 0.05 were considered statistically significant.

Study approval. All animal experiments were approved by the IACUC of the University of Pennsylvania.

\section{Author contributions}

HOR designed and performed experiments and wrote the manuscript. LW performed lung transplantation procedures. JS provided technical assistance. JY assisted with sectioning of slides and immunohistochemistry. MC and LL performed genotyping and assisted with experiments. PA and ZJ provided tissue samples and assistance with preparation of the manuscript. JD provided technical assistance and advice on lung phenotypes. WWH provided assistance with lung transplantation experiments. MLK oversaw the experimental design and writing of the manuscript.

\section{Acknowledgments}

The authors thank members of the University of Pennsylvania Histology Core Facility and the University of Pennsylvania Animal Facility (particularly Latrise Bennett) for their support. We thank Michael Beers, Yaniv Tomer, Meghan Kopp, and Melpo Christofidou-Solomidou at the University of Pennsylvania for their technical expertise and advice. We thank Kunal Patel and John Wherry at the University of Pennsylvania for assistance with cell-tracing experiments. Human lung tissue was provided by the Penn Center for Pulmonary Biology Human Lung Tissue Bank. This work was supported grants from the National Heart Lung and Blood Institute (NHLBI) (T32HL007586-32, to HOR) and the NIH (R01 HL121650, to MLK).

Address correspondence to: Mark Kahn, University of Pennsylvania, Translational Research Center, Room 11-123, 3400 Civic Center Boulevard, Building 421, Philadelphia, Pennsylvania 19104-5159, USA. Phone: 215.898.9007; Email: markkahn@pennmedicine.upenn.edu.
1. Rafii S, Butler JM, Ding BS. Angiocrine functions of organ-specific endothelial cells. Nature. 2016;529(7586):316-325.

2. Jakus $Z$, et al. Lymphatic function is required prenatally for lung inflation at birth. J Exp Med.
2014;211(5):815-826.

3. Mackersie RC, Christensen J, Lewis FR. The role of pulmonary lymphatics in the clearance of hydrostatic pulmonary edema. J Surg Res. 1987;43(6):495-504.
4. Patterson GA, Mitzner WA, Sylvester JT. Assessment of fluid balance in isolated sheep lungs. JAppl Physiol. 1985;58(3):882-891.

5. Rössler A, Fink M, Goswami N, Batzel JJ. Modeling of hyaluronan clearance with application 
to estimation of lymph flow. Physiol Meas. 2011;32(8):1213-1238.

6. Bakocević N, Worbs T, Davalos-Misslitz A, Förster R. T cell-dendritic cell interaction dynamics during the induction of respiratory tolerance and immunity. J Immunol. 2010;184(3):1317-1327.

7. Cook DN, Bottomly K. Innate immune control of pulmonary dendritic cell trafficking. Proc Am Thorac Soc. 2007;4(3):234-239.

8. Stranford S, Ruddle NH. Follicular dendritic cells, conduits, lymphatic vessels, and high endothelial venules in tertiary lymphoid organs: Parallels with lymph node stroma. Front Immunol. 2012;3:350

9. Jones GW, Hill DG, Jones SA. Understanding immune cells in tertiary lymphoid organ development: it is all starting to come together. Front Immunol. 2016;7:401.

10. Aloisi F, Pujol-Borrell R. Lymphoid neogenesis in chronic inflammatory diseases. Nat Rev Immunol. 2006;6(3):205-217.

11. Baluk P, et al. Preferential lymphatic growth in bronchus-associated lymphoid tissue in sustained lung inflammation. Am J Pathol. 2014;184(5):1577-1592.

12. El-Chemaly S, Levine SJ, Moss J. Lymphatics in lung disease. Ann N Y Acad Sci. 2008;1131:195-202.

13. Hess PR, et al. Platelets mediate lymphovenous hemostasis to maintain blood-lymphatic separation throughout life. J Clin Invest. 2014;124(1):273-284.

14. Maby-El Hajjami H, Petrova TV. Developmental and pathological lymphangiogenesis: from models to human disease. Histochem Cell Biol. 2008;130(6):1063-1078.

15. Mislin H. Active contractility of the lymphangion and coordination of lymphangion chains. Experientia. 1976;32(7):820-822.

16. Schmid-Schönbein GW. Microlymphatics and lymph flow. Physiol Rev. 1990;70(4):987-1028.

17. Gashev AA, Davis MJ, Delp MD, Zawieja DC. Regional variations of contractile activity in isolated rat lymphatics. Microcirculation. 2004;11(6):477-492.

18. Gashev AA. Lymphatic vessels: pressure- and flow-dependent regulatory reactions. Ann NY Acad Sci. 2008;1131:100-109.

19. Zawieja DC. Contractile physiology of lymphatics. Lymphat Res Biol. 2009;7(2):87-96.

20. Albelda SM, Hansen-Flaschen JH, Lanken PN, Fishman AP. Effects of increased ventilation on lung lymph flow in unanesthetized sheep. JAppl Physiol. 1986;60(6):2063-2070.

21. Pearse DB, Searcy RM, Mitzner W, Permutt S, Sylvester JT. Effects of tidal volume and respiratory frequency on lung lymph flow. JAppl Physiol. 2005;99(2):556-563.

22. Grimbert FA, Martin D, Parker JC, Taylor AE. Lymph flow during increases in pulmonary blood flow and microvascular pressure in dogs. Am J Physiol. 1988;255(5 Pt 2):H1149-H1155.

23. Choi I, et al. Visualization of lymphatic vessels by Prox1-promoter directed GFP reporter in a bacterial artificial chromosome-based transgenic mouse. Blood. 2011;117(1):362-365.

24. Meinecke AK, et al. Aberrant mural cell recruitment to lymphatic vessels and impaired lym- phatic drainage in a murine model of pulmonary fibrosis. Blood. 2012;119(24):5931-5942.

25. El-Chemaly S, et al. Abnormal lymphangiogenesis in idiopathic pulmonary fibrosis with insights into cellular and molecular mechanisms. Proc Natl Acad Sci U S A. 2009;106(10):3958-3963.

26. Mori M, Andersson CK, Graham GJ, Löfdahl CG, Erjefält JS. Increased number and altered phenotype of lymphatic vessels in peripheral lung compartments of patients with COPD. Respir Res. 2013;14:65

27. Suzuki-Inoue K, et al. Essential in vivo roles of the C-type lectin receptor CLEC-2: embryonic/ neonatal lethality of CLEC-2-deficient mice by blood/lymphatic misconnections and impaired thrombus formation of CLEC-2-deficient platelets. J Biol Chem. 2010;285(32):24494-24507.

28. Bertozzi CC, et al. Platelets regulate lymphatic vascular development through CLEC-2-SLP-76 signaling. Blood. 2010;116(4):661-670.

29. Sweet DT, et al. Lymph flow regulates collecting lymphatic vessel maturation in vivo. J Clin Invest. 2015;125(8):2995-3007.

30. Bénézech C, et al. CLEC-2 is required for development and maintenance of lymph nodes. Blood 2014;123(20):3200-3207.

31. Osada $\mathrm{M}$, et al. Platelet activation receptor CLEC-2 regulates blood/lymphatic vessel separation by inhibiting proliferation, migration, and tube formation of lymphatic endothelial cells. J Biol Chem. 2012;287(26):22241-22252.

32. Hayakawa T, Furusawa T, Yamashita H. A comparative anatomical study of the lymphatic system of the lung in mammals: 5 . Findings in Rodentia. Okajimas Folia Anat Jpn. 1986;63(2-3):73-80.

33. Cui Y, et al. Therapeutic lymphangiogenesis ameliorates established acute lung allograft rejection. J Clin Invest. 2015;125(11):4255-4268.

34. Legge KL, Braciale TJ. Accelerated migration of respiratory dendritic cells to the regional lymph nodes is limited to the early phase of pulmonary infection. Immunity. 2003;18(2):265-277.

35. Vermaelen K, Pauwels R. Accelerated airway dendritic cell maturation, trafficking, and elimination in a mouse model of asthma. Am J Respir Cell Mol Biol. 2003;29(3 Pt 1):405-409.

36. Yadava K, Bollyky P, Lawson MA. The formation and function of tertiary lymphoid follicles in chronic pulmonary inflammation. Immunology. 2016;149(3):262-269.

37. Ager A. High endothelial venules and other blood vessels: critical regulators of lymphoid organ development and function. Front Immunol. 2017;8:45.

38. Acton SE, et al. Podoplanin-rich stromal networks induce dendritic cell motility via activation of the C-type lectin receptor CLEC-2. Immunity. 2012;37(2):276-289.

39. Starling EH. On the absorption of fluids from the connective tissue spaces. J Physiol. 1896;19(4):312-326.

40. Michel CC. Starling: the formulation of his hypothesis of microvascular fluid exchange and its significance after 100 years. Exp Physiol. 1997;82(1):1-30.

41. Bland RD, et al. Lung fluid balance in lambs before and after birth. J Appl Physiol Respir Envi- ron Exerc Physiol. 1982;53(4):992-1004.

42. Sakuma T, Pittet JF, Jayr C, Matthay MA. Alveolar liquid and protein clearance in the absence of blood flow or ventilation in sheep. JAppl Physiol. 1993;74(1):176-185

43. Uhley HN, Leeds SE, Sampson JJ, Friedman M. Role of pulmonary lymphatics in chronic pulmonary edema. Circ Res. 1962;11:966-970.

44. Moessinger AC, Harding R, Adamson TM, Singh M, Kiu GT. Role of lung fluid volume in growth and maturation of the fetal sheep lung. JClin Invest. 1990;86(4):1270-1277.

45. Jennrich S, Lee MH, Lynn RC, Dewberry K, Debes GF. Tissue exit: a novel control point in the accumulation of antigen-specific CD8 T cells in the influenza a virus-infected lung. J Virol. 2012;86(7):3436-3445

46. Buch T, et al. A Cre-inducible diphtheria toxin receptor mediates cell lineage ablation after toxin administration. Nat Methods. 2005;2(6):419-426.

47. Martinez-Corral I, et al. Vegfr3-CreER (T2) mouse, a new genetic tool for targeting the lymphatic system. Angiogenesis. 2016;19(3):433-445.

48. Dashkevich A, et al. Lymph angiogenesis after lung transplantation and relation to acute organ rejection in humans. Ann Thorac Surg. 2010;90(2):406-411.

49. Ruggiero R, et al. Detection of canine allograft lung rejection by pulmonary lymphoscintigraphy. J Thorac Cardiovasc Surg. 1994;108(2):253-258.

50. Bracke KR, et al. Role of CXCL13 in cigarette smoke-induced lymphoid follicle formation and chronic obstructive pulmonary disease. $A m J$ Respir Crit Care Med. 2013;188(3):343-355.

51. Jia J, et al. Cholesterol metabolism promotes B-cell positioning during immune pathogenesis of chronic obstructive pulmonary disease. $E M B O$ Mol Med. 2018;10(5):e8349.

52. Mund SI, Stampanoni M, Schittny JC. Develop mental alveolarization of the mouse lung. Dev Dyn. 2008;237(8):2108-2116.

53. Molet S, et al. Increase in macrophage elastase (MMP-12) in lungs from patients with chronic obstructive pulmonary disease. Inflamm Res. 2005;54(1):31-36.

54. Sandhaus RA, Turino G. Neutrophil elastasemediated lung disease. COPD. 2013; 10(Suppl 1):60-63.

55. Stolz D, et al. Systemic biomarkers of collagen and elastin turnover are associated with clinically relevant outcomes in COPD. Chest. 2017;151(1):47-59.

56. Houghton AM, et al. Elastin fragments drive disease progression in a murine model of emphysema. J Clin Invest. 2006;116(3):753-759.

57. Hunninghake GW, Davidson JM, Rennard S, Szapiel S, Gadek JE, Crystal RG. Elastin fragments attract macrophage precursors to diseased sites in pulmonary emphysema. Science. 1981;212(4497):925-927.

58. Sellami M, et al. Induction and regulation of murine emphysema by elastin peptides. Am J Physiol Lung Cell Mol Physiol. 2016;310(1):L8-23.

59. Hautamaki RD, Kobayashi DK, Senior RM, Shapiro SD. Requirement for macrophage elastase for cigarette smoke-induced emphysema in mice. Science. 1997;277(5334):2002-2004.

60. Demedts IK, et al. Elevated MMP-12 protein lev- 
els in induced sputum from patients with COPD. Thorax. 2006;61(3):196-201.

61. Wong BW, Zecchin A, García-Caballero M, Carmeliet P. Emerging concepts in organspecific lymphatic vessels and metabolic regulation of lymphatic development. Dev Cell. 2018;45(3):289-301.

62. Karkkainen MJ, et al. Vascular endothelial growth factor $\mathrm{C}$ is required for sprouting of the first lymphatic vessels from embryonic veins. Nat Immunol. 2004;5(1):74-80.

63. Huxley VH, Scallan J. Lymphatic fluid: exchange mechanisms and regulation. J Physiol (Lond). 2011;589(Pt 12):2935-2943.

64. Rangel-Moreno J, Hartson L, Navarro C, Gaxiola M, Selman M, Randall TD. Inducible bronchusassociated lymphoid tissue (iBALT) in patients with pulmonary complications of rheumatoid arthritis. JClin Invest. 2006;116(12):3183-3194.

65. Sato M, et al. The role of intrapulmonary de novo lymphoid tissue in obliterative bronchiolitis after lung transplantation. J Immunol. 2009;182(11):7307-7316.

66. Coxson HO, et al. The presence and progression of emphysema in COPD as determined by CT scanning and biomarker expression: a prospective analysis from the ECLIPSE study. Lancet Respir Med. 2013;1(2):129-136.

67. Paris AJ, et al. Neutrophils promote alveolar epithelial regeneration by enhancing type II pneumocyte proliferation in a model of acid-induced acute lung injury. Am J Physiol Lung Cell Mol Physiol. 2016;311(6):L1062-L1075.

68. Wang C, et al. Expansion of hedgehog disrupts mesenchymal identity and induces emphysema phenotype. JClin Invest. 2018;128(10):4343-4358. 